\title{
Papers
}

\section{The long term clinical course of acute deep vein thrombosis of the arm: prospective cohort study}

Paolo Prandoni, Enrico Bernardi, Antonio Marchiori, Anthonie W A Lensing, Martin H Prins, Sabina Villalta, Paola

Bagatella, Donatella Sartor, Andrea Piccioli, Paolo Simioni, Antonio Pagnan, Antonio Girolami

\section{Introduction}

The diagnosis and treatment of deep vein thrombosis of the arm are documented extensively. ${ }^{12}$ The long term clinical course of the condition is, however, poorly defined.

\section{Participants, methods, and results}

Fifty three consecutive patients with a first, symptomatic deep vein thrombosis of the arm, confirmed by ultrasonography or venography-including six cases related to catheter insertionwere treated with high dose heparin, followed by at least three months of warfarin (targeted international normalised ratio 2.03.0) (table).

Follow up visits were scheduled after three and six months, and then every six months up to five years. At each visit, patients underwent a clinical evaluation, for which we used a standardised scale previously validated in patients with venous thrombosis in the leg, ${ }^{3}$ and an ultrasound assessment of the affected venous segments. Each of five symptoms (heaviness, pain, itching, physical limitation, and paraesthesia) and six signs (pretibial oedema, skin induration, discoloration, venous ectasia, redness, and pain during compression) received a score ranging from 0 to 3 . We defined post-thrombotic syndrome as severe in the case of a score higher than 14 and as mild in the case of a score 5-14, on two consecutive examinations. We considered veins as recanalised if they measured less than $2.0 \mathrm{~mm}$ in diameter in a single examination or less than $3.0 \mathrm{~mm}$ in two consecutive examinations at least three months apart.

Symptomatic recurrent thrombosis in the same arm was diagnosed in case of a (new) intraluminal defect on venography, while symptomatic recurrences in other limbs were diagnosed in case the vein could not be compressed on ultrasonography. ${ }^{15}$

We used Kaplan-Meier estimates to assess the risk of recurrent thromboembolism and post-thrombotic syndrome. We used stepwise Cox regression models to calculate hazard ratios for these outcomes in relation to age, sex, extension of thrombosis (single spot versus axillary or subclavian involvement), modality of clinical presentation (idiopathic versus secondary), thrombophilic status, acquired risk factors of thrombosis, and persistent venous obstruction. All patients gave written informed consent.

Two patients were lost to follow up after two and three years, respectively, and 11 died because of cancer progression, pulmonary embolism, and congestive heart failure. ${ }^{1}$ Median follow up was 48.3 months.

Three patients developed a recurrent thromboembolism (recurrence in the same arm in two, and a thrombosis in the leg
Characteristics of the study patients $(n=53)$. Values are numbers (percentages) of patients unless otherwise indicated

\begin{tabular}{lc} 
Characteristics & \\
\hline Mean age in years (SD) & $34.3(18.5)$ \\
\hline Male sex & $4(7.5)$ \\
\hline Location of thrombosis: & $6(11.3)$ \\
\hline Isolated brachial & $14(26.4)$ \\
\hline Axillary vein & $29(54.7)$ \\
\hline Subclavian vein & $6(11.3)$ \\
\hline Axillary and subclavian veins & \\
\hline $\begin{array}{l}\text { Concomitant symptomatic pulmonary } \\
\text { embolism }\end{array}$ & $12(22.6)$ \\
\hline Risk factors for thrombosis & $11(20.7)$ \\
\hline Thrombophilia* & $11(20.7)$ \\
\hline Unusual exercise & $6(11.3)$ \\
\hline Active cancer† & $6(11.3)$ \\
\hline Recent (<4 weeks) trauma or fracture & $3(5.7)$ \\
\hline Previous leg vein thrombosis & $3(5.7)$ \\
\hline Recent (<4 weeks) surgery & $3(5.7)$ \\
\hline Outlet thoracic syndrome & $2(3.8)$ \\
\hline Obesity & $5 / 22(22.7)$ \\
\hline Heart failure & $1 / 22(4.5)$ \\
\hline Oestrogens (\% women) & $9(17.0)$ \\
\hline Pregnancy (\% women) & $8(15.1)$ \\
\hline Combination of inherited and acquired risk \\
factors
\end{tabular}

${ }^{*}$ Factor $\mathrm{V}$ Leiden in six patients, deficiency of protein $\mathrm{C}$ in three, deficiency of antithrombin in teo, and prothrombin gene mutation in one.

†Already known or detected during hospitalisation.

fLonger than six months in four patients.

in one). The cumulative incidence of recurrent thromboembolism after one, two, and five years was 2.0\% (95\% confidence interval 0.0 to 5.9 ), $4.2 \%$ ( 0.0 to 9.9 ), and $7.7 \%$ (0.0 to 16.5 ), respectively. Thirteen patients developed post-thrombotic syndrome, one severely so. Ten cases occurred within six months, two after one year, and one after two years. The cumulative incidence of post-thrombotic syndrome was $20.8 \%$ (9.3 to 32.3 ) at six months, $25.1 \%$ (12.8 to 37.4 ) at one year, and $27.3 \%$ (14.6 to 40.0 )

An additional table showing outcomes in patients with and without vein catheters is on bmj.com 
at two years. It remained stable afterwards. The incidence of these outcomes in patients with and without vein catheter is shown in table A on bmj.com.

Residual thrombosis was related to the incidence of post-thrombotic syndrome (hazard ratio $4.0,1.1$ to 15.0 ). Of other potential risk factors, only thrombosis affecting the axillary and subclavian veins was related, albeit not significantly, to the development of post-thrombotic syndrome (hazard ratio 2.9, 0.8 to 10.7).

\section{Comment}

Symptomatic deep vein thrombosis of the arm carries a low risk of recurrent thromboembolism. Post-thrombotic sequelae occur in almost one fourth of patients within the first two years and are related to residual thrombosis and, to a lesser degree, to the extent of the initial thrombosis. The rates of recurrent thromboembolism and post-thrombotic syndrome are lower than those observed in cohorts of patients with venous thrombosis of the leg. ${ }^{4}$ Whether the incidence of long term sequelae might be further reduced by physical devices or other methods is worth investigating.

Contributors: PP, AWAL, AG, and EB conceived and designed the study. PP, AWLA, MHP, and EB drafted the article. MHP and AM offered their statistical expertise for analysing and interpreting data. PP, SV, PB, DS, AP, and PS provided study patients and collected data by following them prospectively over years. AG, AP, AM, and PS critically reviewed the article for important intellectual content. All authors approved the final version of the article. PP is guarantor.
Funding: None.

Competing interests: None declared.

Ethical approval: Institutional Review Board of Padua University.

1 Prandoni P, Polistena P, Bernardi E, Cogo A, Casara D, Verlato F, et al. Upper extremity deep-vein thrombosis risk factors, diagnosis, and complications. Arch Int Med 1997;157:57-62.

2 Kommareddy A, Zaroukian MH, Hassouna HI. Upper extremity deep venous thrombosis. Semin Thromb Hemost 2002;28:89-99.

3 Prandoni P, Lensing AWA, Cogo A, Cuppini S, Villalta S, Carta M, et al. The long-term clinical course of acute deep venous thrombosis. Ann Intern Med 1996;125:1-7.

4 Kahn SR, Hirsch A, Shrier I. Effect of post-thrombotic syndrome on health-related quality of life after deep venous thrombosis. Arch Intern Med 2002;162:1144-8.

5 Hirsh J, Lee AY. How we diagnose and treat deep vein thrombosis. Blood 2002;99:310210.

doi $10.1136 /$ bmj.38167.684444.3A

Department of Medical and Surgical Sciences, 2nd Chair of Internal Medicine, University Hospital of Padua, Via Ospedale Civile 105, 35128 Padua, Italy

Paolo Prandoni associate professor

Enrico Bernardi medical doctor

Antonio Marchiori medical doctor

Sabina Villalta medical doctor

Paola Bagatella medical doctor

Donatella Sartor medical doctor

Andrea Piccioli medical doctor

Paolo Simioni assistant professor

Antonio Pagnan professor

Antonio Girolami professor

Center for Vascular Medicine, Academic Medical Center, University of Amsterdam, Meibergdreef 9, 1105 AZ Amsterdam, Netherlands

Anthonie WA Lensing medical doctor

Department of Clinical Epidemiology and Technology Assessment, University of Maastricht, PO Box 5800, 6202 AZ Maastricht, Netherlands

Martin H Prins professor

Correspondence to: P Prandoni paoloprandoni@tin.it 